\title{
REYES Y NOBLES EN LA ICONOGRAFIA FRANCISCANA
}

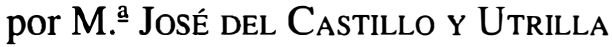

La Iconografía de la Orden de Friles Menores (O.F.M.) de San Francisco, presenta uno de los panoramas iconográficos más ricos de las Ordenes religiosas.

Como ejemplo tomamos a los Santos Reyes y Nobles que ellos toman como Patronos de sus Provincias. De estos personajes, unos pertenecieron a la orden, como son: Santa Isabel Reyna de Hungría; San Luis Rey de Francia, ambos Terciarios Franciscanos, y San Luis Obispo de Tolosa, Príncipe de Anjou, y heredero de la Corona de Nápoles, que fue fraile franciscano.

Otro grupo de estos Reyes y Nobles, lo constituyen aquellos Santos, que sin tener relación con la O.F.M., eran considerados como Patronos de algunas de las Provincias que los Franciscanos tomaron bajo su custodia. Entre ellos, y hasta el S. XVI, contamos con: Santa Ursula, Princesa de Colonia; San Carlomagno, Emperador de Europa; San Leopoldo, Duque de Austria, y San Venceslao, Duque de Bohemia.

Razones históricas unas veces, propagandísticas otras, pero siempre la adecuación de los franciscanos a los territorios en que se establecían y el imperativo de no romper con las tradiciones del país, se muestran en estos ejemplos.

The iconography of the Order of Friars Minor of Saint Francis offers one of the richest pictorial panoramas of all the religious orders.

By way of example we consider the Holy Kings and Nobles adopted by the Order as Patrons of their Provinces. Some of these individuals actually belonged to the Order, such as St. Elizabeth, Queen of Hungary and St. Louis, King of France, both Franciscan Tertiaries, and St. Louis, Bishop of Toulouse, Prince of Anjou and heir to the Crown of Naples, who was a Franciscan friar.

Another group of these Kings and Nobles is formed by those Saints who, while not belonging to the Order, were considered Patrons of some of the Provinces taken under the custody of the Franciscans. Among them, down to the 16th century, are: St. Ursula, Princess of Cologne; St. Charlemagne, Emperor of Europe; St. Leopold, Duke of Austria, and St. Wenceslas, Duke of Bohemia.

These examples reflect historical motives in some cases, reasons of propaganda in others, but always the adaptation of the Franciscans to the territories in which they were newly-established, and the imperious need not to break with the traditions of the country. 
El panorama iconográfico que nos presenta el Franciscanismo es tan amplio, que es fácil perderse dentro de él.

Los símbolos, los emblemas, la pura historia de la Orden y las representaciones de sus más distinguidos miembros, nos pueden llevar, con facilidad, a un mundo de confusión, en el que aparentemente, no se encuentra claro el hilo conductor, y en el que las interpretaciones pueden, y de hecho así sucede, antojársenos imprecisas.

Pero si abordamos el tema con serenidad, y sin quererlo abarcar de una manera exhaustiva $y$, sobre todo, apresurada, podremos, con una relativa facilidad, encontrar las razones, además de las justificaciones históricas y religiosas, por las cuales estas representaciones iconográficas no van a circunscribirse al exclusivo ámbito de la O.F.M., y de sus Santos más representativos, sino que comprenderán también a otros, que sin que hubieran tenido la menor relación con los Franciscanos, son adoptados por éstos, como Patronos de las Provincias de esta Orden Mendicante.

Como un reflejo de este hecho, fácil de comprobar, es la titulación de una serie de Reyes y Nobles, como Patronos Franciscanos. Al análisis de este fenómeno voy a dedicar las pocas páginas que continúan.

De entre los siete ejemplos sometidos a estudio, solamente tres tienen una relación directa con el franciscanismo, son: San Luis Rey de Francia; Santa Isabel, Reyna de Hungría y San Luis de Anjou, Obispo de Tolosa.

Los otros cuatro: San Carlomagno; San Leopoldo de Austria; Santa Ursula de Colonia y San Venceslao de Bohemia, son ajenos a la Orden.

Existe una cuestión muy importante como objeto de reflexión en lo que la O.F.M. se refiere, en el aspecto que atañe a la asimilación de tradiciones locales y devociones ancestrales de determinados territorios, acoplados a sus proyectos de evangelización.

Los cuatro Santos, Reyes y Nobles, que no corresponden al Santoral estrictamente Franciscano, están tomados, en directo, de la tradición devota popular.

Comenzamos nuestro comentario por aquellos personajes que sin pertenecer de ninguna manera a la O.F.M., tuvieron en sus actividades, algunas connotaciones afines a la Orden ,y sobre todo, aquellos que por sus virtudes evangélicas, destacaron de alguna manera que pudiera asimilarse a los postulados franciscanos.

También en su incorporación a la larga fila de los venerados, contó la tradición de los pueblos que incluso con anterioridad a la aparición del franciscanismo, habían hecho de ellos casi héroes nacionales, en versión histórico-santa.

El amplio proceso de reorganización de la Orden Franciscana, que tuvo lugar en el S. XVI, y que el General de la misma, Fray Francisco Gonzaga recopiló en 1587, hizo que la distribución de los antiguos territorios concedidos a la exten- 
sión del franciscanismo, se delimitaran en Provincias hasta el momento inexistentes, o consideradas como Custodias dependientes de los centros más importantes.

Es entonces, en el S. XVI, cuando comienzan a aparecer como Patronos los Santos Nacionales, aunque no todos los casos que citaremos corresponden estrictamente a esa centuria. Hay algunos que habían sido institucionalizados con anterioridad, y precisamente, apoyándose los franciscanos en la tradición establecida y en la devoción del pueblo, evitan imponer un nuevo santoral que sustituyera al ancestral. Esto por supuesto no implica por parte de la Orden, el que no convivan los santos locales con los propios del santoral franciscano.

Es frecuentísimo que los patronos de los conventos de cada una de las provincias, sean, sobre todo, San Francisco; San Pedro y San Pablo; San Bernardino; La Concepción; San Juan Bautista, etc..., todos ellos comunes a las distintas Provincias Franciscanas, en todos los Continentes.

Junto a los citados, aparecen otros propios de cada territorio o jurisdicción, como son: San Aegildo; San Vito y San Modesto; Los Santos Protectores de Cadani (En Bohemia); Santa Gertrudis, etc.

Los Santos Reyes y Nobles de los que vamos a ocuparnos, tenían bajo su patrocinio las siguientes Provincias: San Luis Rey de Francia, la de Francia, que correspondía territorialmente a la Isla de Francia, San Luis de Anjou, Obispo de Tolosa, la de Tolosa, que comprendía casi todo el Sur de Francia; Santa Isabel, Reyna de Hungría, la de Santa María de Hungría, en la que entraban, además de territorios húngaros, algunos pertenecientes a la actual Austria; San Carlomagno, la de Sajonia; teniendo también fundaciones en otras regiones del Centro de Alemania; San Leopoldo de Austria, la del Tirol, y se extendía por el Suroeste de Alemania, y San Venceslao, la de Bohemia, repartida en una gran parte de la actual Checoslovaquia.

El primer grupo de personajes del que vamos a ocuparnos, es el que tiene en si una relación directa con la Orden Franciscana.

Todos ellos, significan de alguna manera las virtudes básicas de los postulados de los seguidores del Santo de Asís: la Humildad, la Caridad y la Evangelización.

Independientemente de la categoría de cada uno de los Santos, todos ellos coinciden en sus actuaciones, con los matices diferenciadores lógicos.

La iconografía que aquí presentamos está tomada de la ya referida obra de F. Francisco Gonzaga, y el autor de los grabados es Giovanni Batista Crespi.

$\mathrm{La}$ traza de las orlas que encierran a las figuras, responde al estilo manierista. Son muy cuidadas, a base de guirnaldas, tarjas, medallones y demás elementos característicos. Los modelos, parecidos entre si, en ocasiones se completan con algún símbolo relativo a la personalidad a la que enmarcan, como es en el caso 
de Santa Ursula; (Fig. 6) que en su remate tiene la figura de dos querubines que sostienen la corona y la palma del martirio alusivas a la historia de la Santa.

El orden por el que vamos a analizar a cada grupo, no es caprichoso. Se sigue el orden de canonización de cada uno de los santos, en cada uno de los grupos en que están divididos: El primero, de los pertenecientes a la Orden. El segundo, el de los asimilados a ella.

El primer lugar, según este criterio, le corresponde a Santa Isabel de Hungría, que fue canonizada en Perugia en 1235. La vida y la historia de Isabel no se remontan a mucho tiempo atrás, pues, hija del Rey Andrés II de Hungría, nace en 1207 y muere en 1231.

Su historia, aunque muy conocida, vamos a resumirla en unos pocos trazos, para facilitar así la comprensión de la manera de estar representada.

Dadas ya las fechas de su nacimiento y su muerte, otros dos datos importantes en su corta vida, son los de su matrimonio en 1221 con el duque de Turingia, y el de su viudez y retiro voluntario a Marburg, en 1227. Junto a su castillo de Wartburg funda un hospital para pobres, acogiendo en él especialmente a los leprosos, de los que cuida personalmente.

En el mismo año de 1227, toma el hábito de la Orden Tercera de San Francisco, y en los pocos años que pasaron desde este retiro voluntario y su fallecimiento, alternó la oración y el ascetismo, con el ejercicio de la caridad.

Los ciclos de sus representaciones son varios, pero los más conocidos son los que corresponden a la llamada "Caridad de Santa Isabel", en los que aparece cuidando a los tiñosos, el de la transformación del leproso en Cristo, y sobre todo, "El milagro de las Rosas", que es el pasaje más difundido de su vida, desde el punto de vista iconográfico.

A este "Milagro de las Rosas", pertenece la representación que hace Crespi en la Crónica Franciscana.

Santa Isabel va vestida con el hábito franciscano, ceñido con el cordón; su cabeza está cubierta con las tocas y rostrillo de viuda, y porta la corona real que ciñe sus sienes. Con ambas manos sujeta abierto el lienzo lleno de rosas. Las rosas blancas y rojas en que se habían convertido los panes de la caridad.

Como decíamos, esta representación es quizás la más clásica de cuantas atañen a la iconografía de esta Santa. (Fig. 1).

Su devoción se extendió rápidamente, promovida por los Cistercienses, que establecen su fiesta en 1236, y por los Dominicos, pero quienes más contribuyeron a la difusión de su culto, fueron los Franciscanos y los Caballeros de la Orden Teutónica, que la hacen su Patrona.

Inmediatamente después de la reorganización de las Provincias y Custodias Franciscanas, la antigua demarcación de Santa María de Hungría, se acoge bajo el patrocinio de Santa Isabel, sin perder no obstante su primera denominación. 
El segundo, según la cronología establecida, es San Luis, Rey de Francia, canonizado por Bonifacio VIII en 1297. Este, como Santa Isabel, tiene una historia inmediata a la fecha de su subida a los altares, ya que nace en 1215 , es coronado Rey en 1226 y muere de peste en el cerco de Túnez, en 1270, no sin antes haber estado prisionero en Damieta, en 1248. A la vuelta de la Cruzada, ingresó como Terciario en la Orden de San Francisco, y sus relaciones con esta, y en especial con Fray Elias, uno de los más insignes discípulos directos del Santo de Asís, son de sobra conocidas.

Las historias y leyendas de las relaciones del Monarca con la O.F.M., se cuentan en la mayoría de las Crónicas Franciscanas, desde la de Tomás de Celano, hasta la de los "Compañeros".

No obstante, su biografía no se encuentra reseñada en la famosa Leyenda Aurea, del Domínico Jacobo de la Voragine, sino que los autores que la difunden son Guillermo de Saint-Pathus, confesor de la Reyna Margarita, esposa de Luis, y por las Crónicas de Joinville.

Durante la Edad Media, difícilmente lo vemos aparecer con los atributos reales. Sus primeras iconografías nos lo representan como un segundo San Francisco, vestido con el hábito de la Orden y el cordón característico, pero siempre coronado con el símbolo de la realeza.

Las escenas en las que se le representan, son varias, siempre relacionadas con el ejercicio de la caridad y la evangelización. Así, aparece dándole de comer a los enfermos, o bien lavando los pies a los pobres. También en muchos casos, aunque aparezca con los atributos reales, sobre todo a partir del $\mathrm{S}$. $\mathrm{XV}$, suele mantener en sus manos los Clavos de Cristo y la Corona de Espinas, com testimonio de su voluntad de rescatar los Santos Lugares y su participación en las Cruzadas.

A su muerte, su hermano Carlos de Anjou, hizo enterrar su corazón en la Abadía de Monreale, cerca de Palermo, mientras que su cuerpo se trasladó a la Abadía de San Denis, Panteón Real de la Monarquía Francesa.

Más tarde, en 1306, bajo el Reinado de Felipe el Hermoso, su féretro es trasladado a la Saint-Chapelle, que se había construido para guardar la Corona de Espinas, reliquia donada por San Luis.

Patrón de París y de Poissy, su villa natal, su culto se extiende por Francia muy rápidamente, aunque no es considerado como patrón de la Casa Real francesa, hasta el S. XVII.

Además de en Francia, su veneración se extendió desde el S. XIV por Italia, promovida tanto por los Franciscanos como por la Casa de Anjou, entonces reinante en Nápoles.

Aquí aparece vistiendo el hábito franciscano, ceñido con el cordón, y como símbolos de su condición de Rey, lleva el cetro con la flor de lis, la capilla de armiño y la corona. (Fig. 2). 
El tercero de la serie es San Luis de Anjou, Obispo de Tolosa, Patrón de la Provincia de San Luis, que como tenía su centro en Tolosa y comprendía gran parte del Sur de Francia.

Este personaje, que fue canonizado en 1317, había nacido en 1274. Era el hijo mayor del Rey de Nápoles, Carlos II de Anjou y de María de Hungría, era descendiente por tanto de San Luis y de Santa Isabel.

Fue educado por los franciscanos en Barcelona y renuncia a la corona de Nápoles, a favor de su hermano Roberto, para ingresar en la Orden de San Francisco, en 1296. Fue consagrado Obispo por el Papa Bonifacio VIII en Febrero de 1297 y en el mismo año, el 9 de Agosto, falleció.

Se entierra en la iglesia franciscana de Les Cordeliers de Marsella, y su culto se extiende rápidamente por Francia e Italia, propiciado tanto por su Orden, como por su hermano Roberto que ya reinaba en Nápoles.

Sus primeras representaciones datan de la fecha de su canonización, y su inoconografía es muy regular, apareciendo generalmente con los atavios de Obispo, pero con la capa flordelisada como símbolo de su rango familiar, y la corona a sus pies y sosteniendo el libro del confesor. (Fig. 3).

No obstante se conocen de él varios ciclos de su vida, uno debido a Simone Martini, que es el que se encuentra en la predela del Exvoto de Roberto de Anjou, en la iglesia de San Lorenzo el Mayor de Nápoles, y otro que es el que representan los frescos de Ambrosio Lorenzetti, en el refectorio del convento franciscano de Siena, encargados por la Comunidad en 1331.

Si estos tres Santos que anteceden, como hemos visto, tenían una relación directa con la O.F.M., de los que trataremos a continuación no se puede decir lo mismo, aunque como ya advertíamos al principio, fueron adoptados por ella, debido generalmente a causas tradicionales.

El primero de todos los cuatro que siguen, es Carlomagno, venerado como Santo durante un largo tiempo y en él concurren una serie de circunstancias bastante curiosas, que hacen de su figura un caso excepcional dentro de la hagiografía.

Nace el Emperador de Europa en el año 742 y muere en el 814. Su biografía está repleta de azañas y no es necesario comentar su trayectoria histórica, de sobra conocida. Coronado Emperador del Sacro Imperio romano Germánico, en el año 800, por León III, Europa fue su reino, hasta el punto de que en algunas crónicas medievales, toda Centro-Europa, Francia y parte de Italia son conocidas como "El Reino de Carlos"..

En el año 1165, el 29 de Diciembre, fue canonizado por Pascual III, a instancias del Emperador Federico Barbarroja, pero como el Pontífice tenía problemas entonces con la Curia Romana, y se le consideró Antipapa, la canonización de Carlomagno nunca fue refrendada por la Santa Sede. 
No obstante estos litigios internos de la Curia y el Papado, la personalidad del Emperador de la "Barba Florida", trasciende más allá de la simple historia oficial. Verdaderamente, ya nos basemos en la historia o en la leyenda, en ambas podemos encontrar bases suficientes com para magnificar a este personaje.

Sus representaciones figurativas no son muy abundantes, podemos recordar la pequeña estatua ecuestre de Metz, no obstante, su historia, está más extendida, y los romances medievales, tienen en ella una de las fuentes más fértiles y un motivo de inspiración insuperable.

Difundieron sus hazañas y su vida los monjes benedictinos, a los que siempre había protegido e incluso, encomendado en parte el gobierno de sus reinos. Así, el monje Eginardo en primer lugar, y después, en el S. XII, el Arzobispo de Reims, Turpin, contribuyeron no poco a popularizar algunos pasajes de los más significativos de la historia de Carlomagno.

Sus ciclos históricos corresponden a tres aspectos simbólicos aún apoyándose en la realidad. Por una parte, lo encontramos como peregrino a Constantinopla, aunque sea a través de un sueño en el que aparece Constantino, entregándole una serie de reliquias para que sean veneradas en sus reinos. En estos pasajes se puede interpretar el simbolismo místico de la historia del Emperador.

El segundo pasaje importante de los ciclos carolingios, es el de la Cruzada de España, cantada tanto en la literatura medieval inglesa, como en la trova francesa, sin olvidarnos, por supuesto, de los romances castellanos. El episodio de Roncesvalles y las lanzas floridas, son temas muy conocidos, pero significan a su vez la defensa de la Cristiandad, la defensa del Papado y en consecuencia, el iniciador de las Cruzadas, si bien históricamente esto es falso.

El último de los hechos representativos es "La Misa de Saint Gilles", que simboliza la penitencia, a la vez que el perdón de un pecado de incesto, cometido por el Emperador con su hermana Berta, del que nació el famoso Roldán.

A pesar de que como dijimos, la canonización de Carlomagno no fue refrendada por Roma, no se prohibió su culto, que se extendió por Francia, Alemania, Cataluña, Holanda y Suiza.

El centro de las devociones es Aiz-la-Chapell, donde está su enterramiento, mandado hacer por Federico II. En el sarcófago que contiene los restos, aparecen representadas escenas de su vida, según la versión de la crónica de Trupin.

Cuando su culto adquiere mayor importancia es en el S. XIV, en Alemania durante el reinado de Carlos IV, y en el S. XV en Francia, con Luis XI, que en 1475 instituye fiesta nacional el 29 de Enero, fecha conmemorativa de la canonización, y lo nombra, junto con San Denis y San Luis, patrón de la casa real de Francia.

Aunque las historias y leyendas concernientes a Carlomagno son abundantes y sugestivas, la manera más frecuente de estar representado es la que aquí pre- 
sentamos (Fig. 4), con los atributos del Imperio; Coronado, con la espada en una mano y en la otra, el globo terráqueo, rematado con la cruz, símbolos que aparecen como exponentes máximos de la unión de la Iglesia y el Estado, que cristalizó en el Sacro Imperio Romano Germánico.

Continuamos ahora con San Leopoldo, Patrón de la Provincia de Tirol. Nacido en Melk, junto al Danubio, en 1073, muere en 1136, y es canonizado en 1485.

Margrave de Austria, es miembro de la dinastía de los Babemberg, antecesora de los Habsburgo y se casa con Inés, hija del Emperador Enrique IV de Alemania, con la que tiene la nada despreciable prole de dieciocho hijos.

Se le conoce sobre todo, en el aspecto religioso, como confesor y fundador, siendo la más importante de las que realiza, la de los Agustinos de Kosterneuburg, cerca de su residencia de Leopoldsberg, en los alrededores de Viena. Esta fundación, también tiene su leyenda, la conocida como "Leyenda del Velo", en la que está implicada una aparición milagrosa de la Santísima Virgen.

Los franciscanos lo adoptan como Patrón de la Provincia del Tirol en 1560, debido a la gran devoción que hacía San Leopoldo tenían los Austriacos, pero no es hasta 1663, cuando es proclamado como patrono de la Baja Austria.

No hay demasiadas representaciones de los ciclos de San Leopoldo, por eso su versión más conocida es en la que aparece con traje militar, manto y corona y en su mano, el cetro. (Fig. 5).

Los dos santos que continúan en la relación de los Nobles adoptados como patronos por los franciscanos, se encuentran Santa Ursula, por la Provincia de Colonia y San Venceslao, por la de Bohemia.

Ninguno de los dos tiene una referencia documental de la fecha de su canonización ni de su nacimiento, pero refiriéndonos a la historia de cada uno de ellos, podemos comprobar que la de Santa Ursula es aún más antigua que la de San Venceslao, puesto que la tradición de la primera corresponde a tiempos inmemoriales.

$\mathrm{Su}$ historia está conectada con la de las Once mil Vírgenes, y se narra por primera vez en la "Historia de los Reyes de Britania", debida a Geodffroy de Monmuunth. Historia que más tarde fue ampliada por Hermann-Joseph de Steingfeld y por la Abadesa visionaria Elisabeth de Schönhau.

La aparición de Ursula, hija del Rey de Gran Bretaña (no sabemos cual), como patrona de las vírgenes, se remonta al S. XI, aunque anteriormente, en Colonia y su región, este privilegio lo ostentaba Pinnose, patrona del monasterio de Essen.

Cuando en el S. XI los restos de Pinnose fueron trasladados desde el cementerio de "ager Ursulanus", al monasterio del que fue patrona, los coloneses cambiaron su nombre por el de Ursula, que es como se conoce a partir de entonces.

La historia de Santa Ursula y las Oncemil Vírgenes, en su versión más 
conocida, es la que nos proporciona la Leyenda Dorada de Jacobo de la Voragine, donde nos cuenta su matrimonio con un príncipe pagano, la conversión de éste al cristianismo, la peregrinación a Roma con todo el séquito de las doncellas, el viaje de vuelta a la ciudad alemana y la muerte de todos los miembros de la comitiva a manos de los Hunos a las puertas de Colonia.

Aunque la historia de la Santa está muy difundida, y en los ciclos de su vida la vemos representada en múltiples ocasiones, sin faltarnos la tan repetida de su entrevista con el Papa Ciriaco y su viaje por el Rin, la manera más común de todas es la que presenta a Ursula con los símbolos de su martirio, concretamente, con la flecha atravesándole el cuello, la palma y el estandarte con la Cruz, que - fue el signo por medio del que convocó a sus compañeras para que se mantuvieran firmes en la Fe.

Así es como se representa aquí (Fig. 6), adornada además con la corona que denota la nobleza de su linaje.

Por último nos encontramos con San Venceslao, Duque de Bohemia, Patrón de esta Provincia Checoslovaca. No se conoce de él la fecha de su nacimiento, ni de su canonización, aunque si la de su muerte, que acaeció en el año 935.

Fue San Venceslao el primer príncipe checo convertido al Cristianismo, religión en la que le había educado su abuela. La corte le era contraria, y sobre todo, su hermano Boleslas, quien para evitar que se propagaran las nuevas creencias, lo mandó asesinar.

Reconocido como Patrón de Bohemia en le S. XI, sus reliquias se conservan en la iglesia de San Gui de Praga.

Significan las representaciones de este personaje, la evangelización de su pueblo. Normalmente aparece vestido con el traje militar, el bonete con la corona ducal, que denotan su rango. En una de sus manos lleva el estandarte con el águila imperial y en la otra la palma signo del martirio. (Fig. 7).

El culto de las reliquias de San Venceslao y de su memoria, se extendió rápidamente por Checoslovaquia e incluso por parte del Norte de Austria, donde su figura, en ocasiones. aparece como un paralelo de San Leopoldo.

Estas siete figuras que apuntamos, son las más representativas que en la Orden de San Francisco encontramos como miembros de la alta nobleza, aunque, por supuesto, no son las únicas.

Hemos tomado estos ejemplos, por considerarlos "oficiales", ya que aparecen con sus respectivos patronazgos provinciales, estén los personajes vinculados o no directamente con la O.F.M.

Otros nombres, como los de Santa Isabel de Portugal, fundadora de las Clarisas de Coimbra; San Ivo de Burdeos, el mismo Fernando III, se han puesto en relación en ocasiones con la Orden de San Francisco, si bien los testimonios históricos no dan noticias directas sobre este aspecto. 
Es frecuente, por supuesto, que sobre todo en el tiempo que va desde el S. XIII hasta el S. XVI, la labor de estos Frailes Menores, reclutó a miembros muy diversos de las distintas capas de la sociedad para su obra, pero el fenómeno más evidente, es que en el S. XVI, cuando la Reforma del Cardenal Cisneros, para los franciscanos españoles, y la Reorganización de la orden en general por fray Francisco Gonzaga, que fue quien mandó hacer la recopilación en la que nos basamos, se atribuyeron nuevos patronazgos a muchas Provincias y para ello se recurrió como ya hemos visto, por ejemplo en el caso de la de Sajonia y de la de Hungría, a Santos nacionales, pertenecientes a la monarquía de cada país.

Este fenómeno es lógico históricamente, ya que en S. XVI, la tendencia a las monarquías absolutas y a la exaltación de las mismas es un hecho que de alguna forma también influye en la Iglesia y de ahí esa adopción de los personajes nobles como patronos, para de alguna manera enaltecer también a la propia Orden, a la vez que por ese camino, solidarizarse aún más con los territorios en los que las Provincias Franciscanas estaban establecidas.

\section{BIBLIOGRAFIA}

La bibliografía que reseñamos a continuación atañe de una manera relativa al tema que nos ocupa. Como se comprobará en el texto no hemos incluido citas ni hacemos referencia a notas por considerarlas innecesarias para la aclaración del mismo, aunque si es conveniente reflejar las obras que nos han servido de guía para la redacción de este trabajo.

BERLINER, Rudolf: Modelos Ornamentales de los Siglos XV al XVIII. Barcelona. s / $\mathrm{f}$.

CASTILLO Y UTRILLA, María José del: Posibles influencias de una Crónica franciscana en la temática de Murillo.

Rv. "Archivo Hispalense" n.․ 195. Sevilla 1981.

GONZAGA, Fr. Francisco: De Origine Seraphica Religionis.

Imp. D. Basa. Roma 1587

HIND, Arthur M.: Early Italian Engraving.

Rp. Leichtenstein 1970.

REAU, Luis.: L'Iconographie de l'art Cheretienne.

VI. I, II, II. (3) P.U.F. parís 1958.

VORAGINE, Jacobo de la : Leyenda Dorada.

Alianza Forma. Madrid 1982.

WAGNER, Klaus: Los autores franciscanos en la desaparecida biblioteca del Convento de San Francisco.

Rv. "Archivo Hispalense". n. ${ }^{9}$ 192. Sevilla 1980.

YARZA, Joaquín et alt:: Fuentes Documentales para la Historia del Arte. Gustavo Gili. Barcelona 1982. Vl. II y III. 


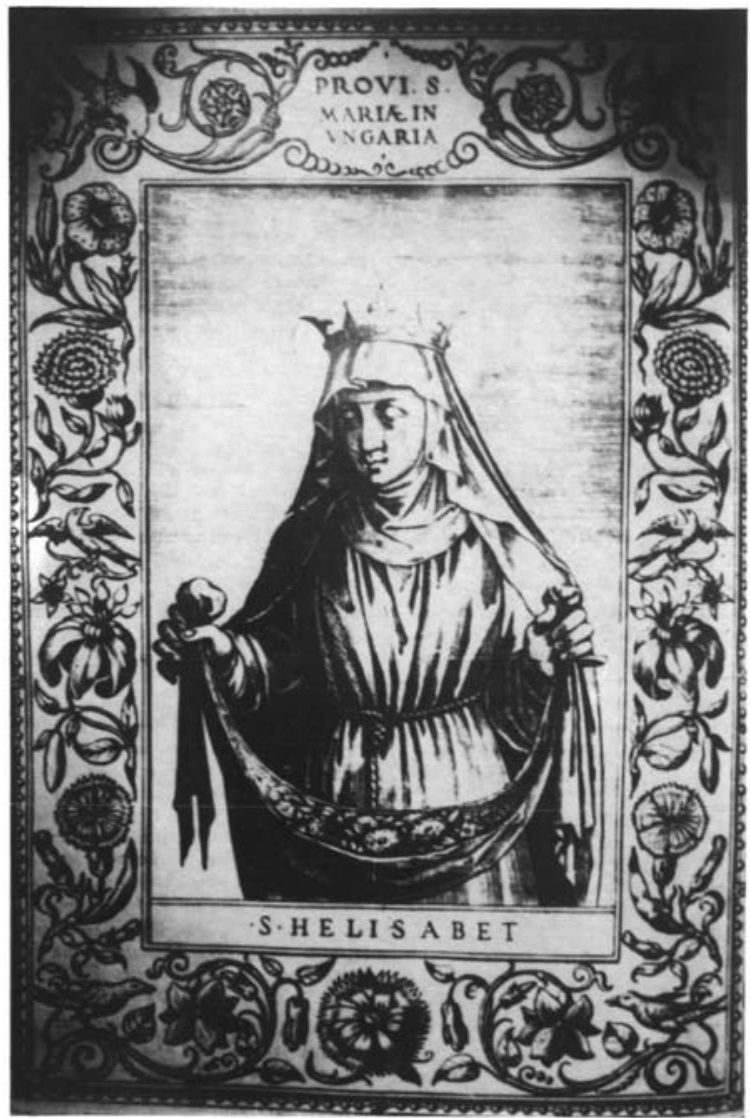

Fig. 1. Santa Isabel, Reyna de Hungria. Terciaria Franciscana. † 1231, - cn. 1235. 


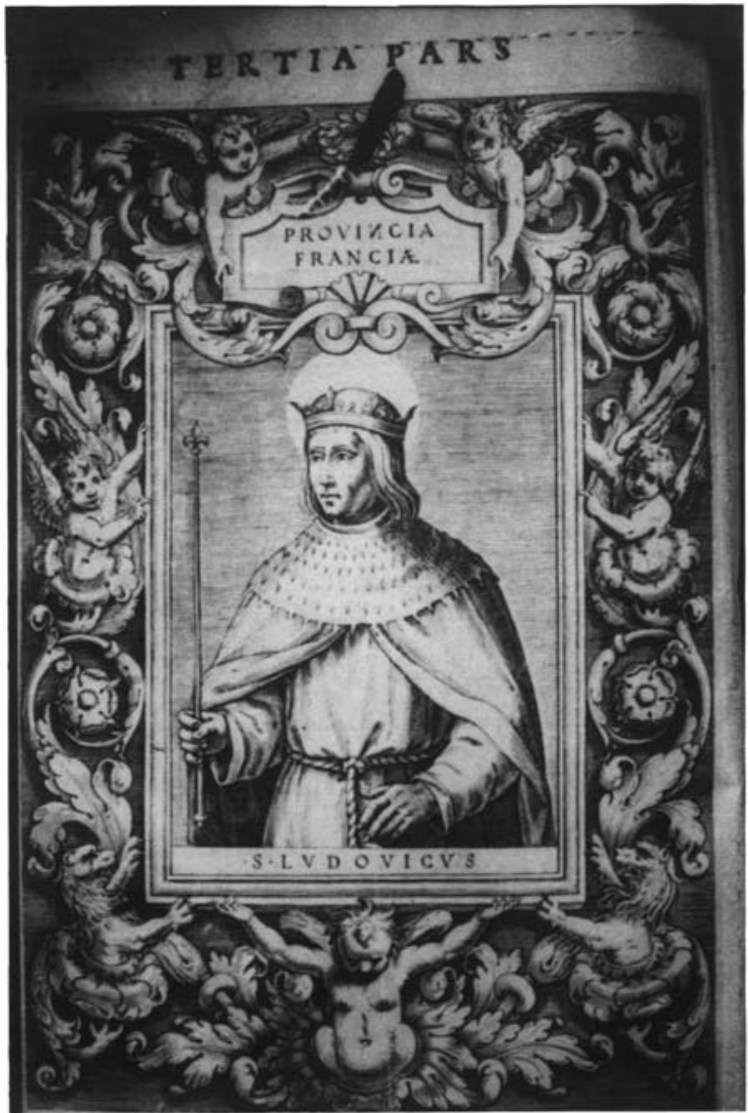

Fig. 2. San Luis Rey de Francia Terciario Franciscano. † 1270.- cn. 1297 


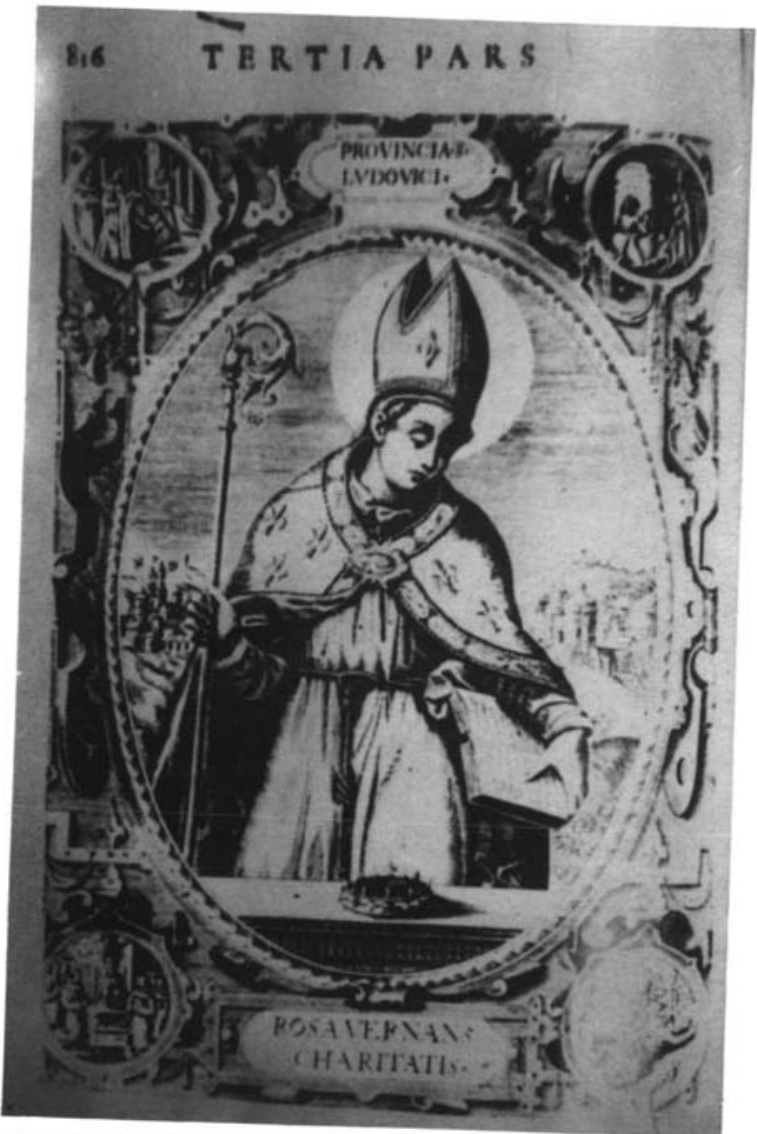

Fig. 3. San Luis de Anjou. Obispo de Tolosa. Fraile Franciscano. † 1297. - cn. 1317. 


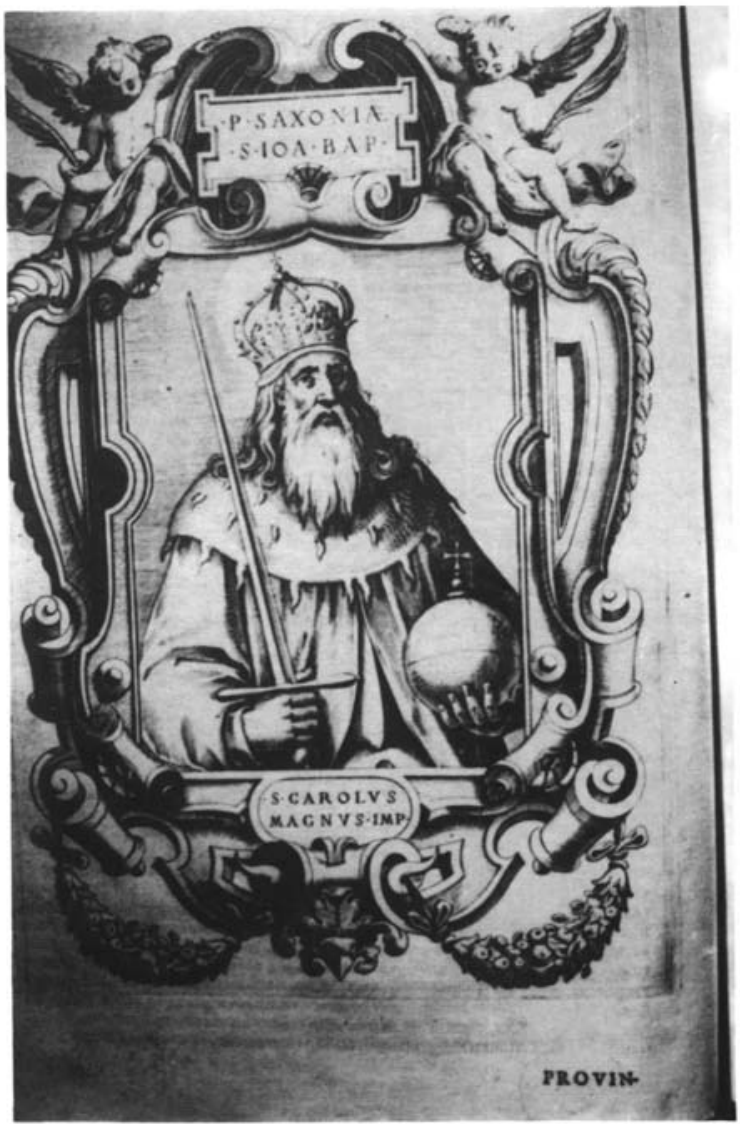

Fig. 4. San Carlomagno Emperador. +814.- cn. 1165. 


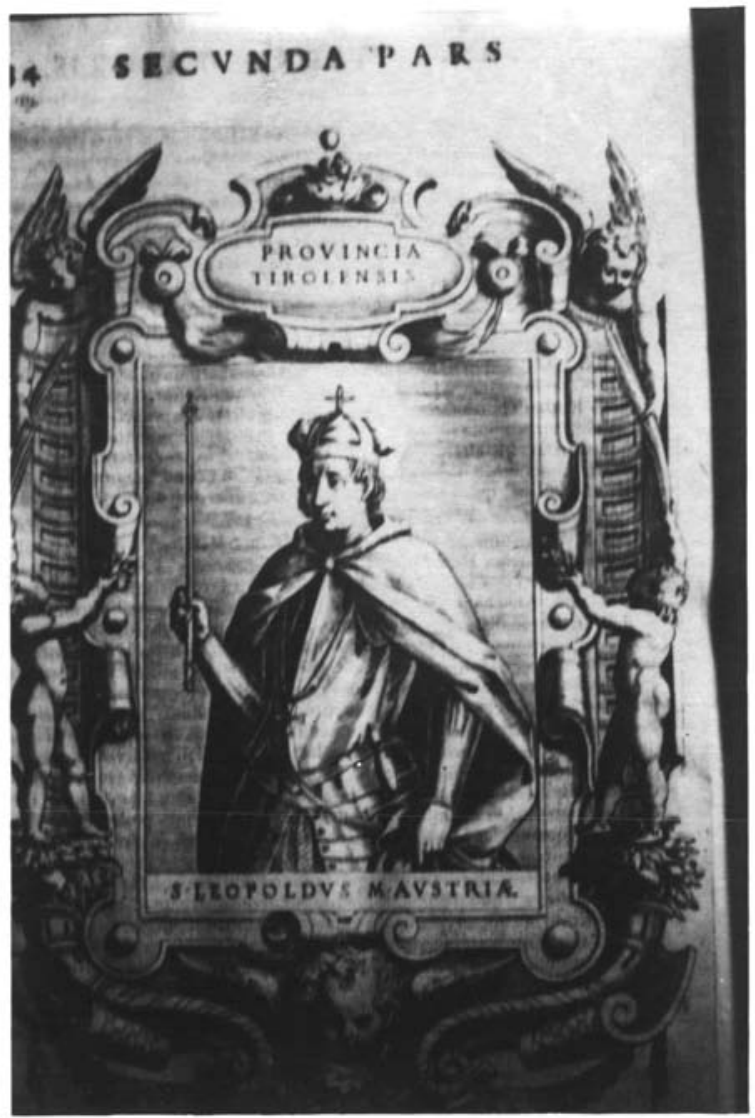

Fig. 5. San Leopoldo Madgrave de Austria. $+1136 .-\mathrm{cn} .1485$ 


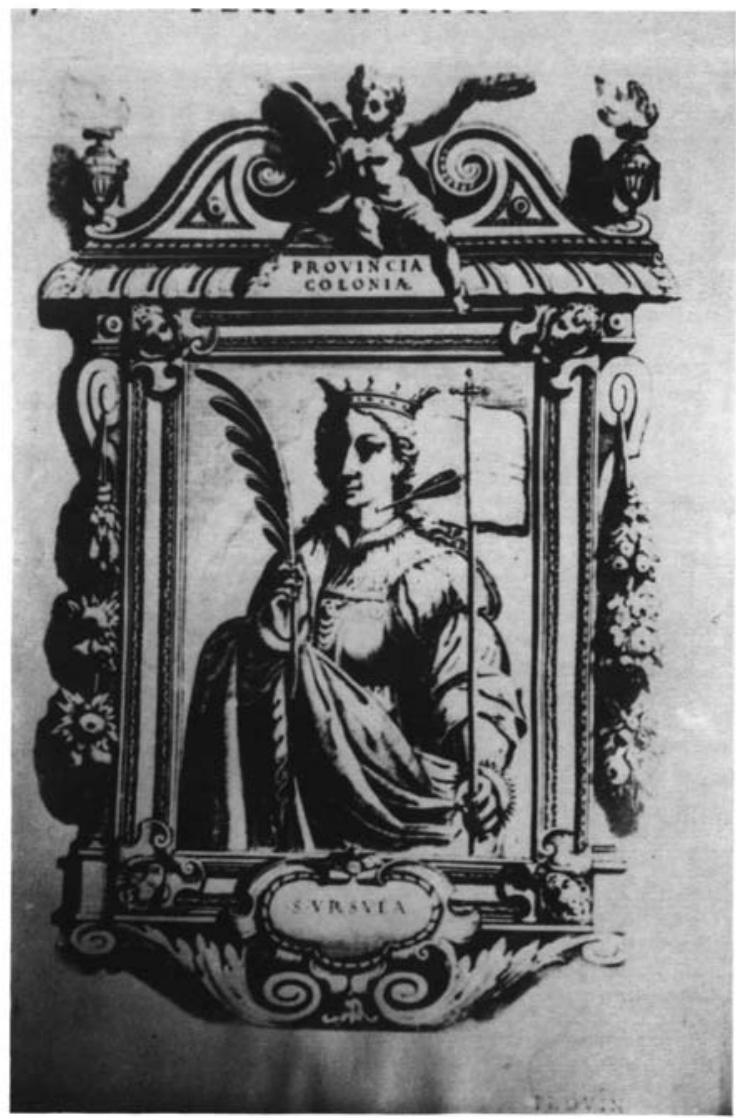

Fig. 6. Santa Ursula, Princesa de Colonia y Bretaña. Sin datos concretos. S. XI c. 


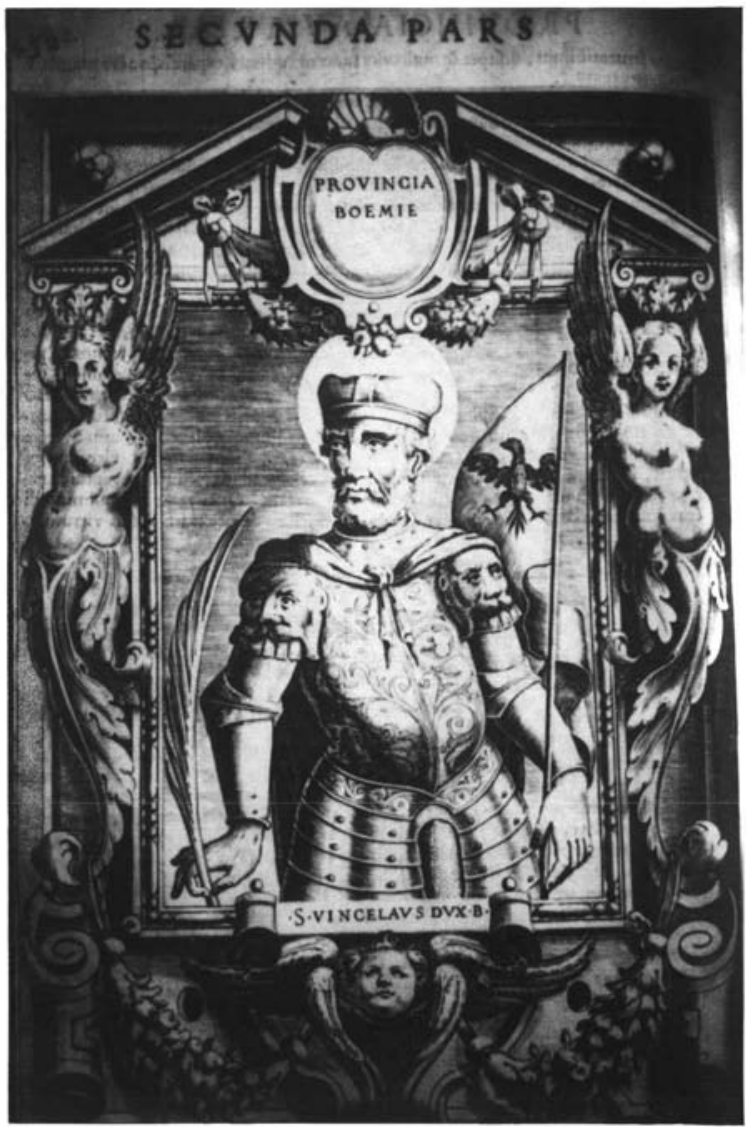

Fig. 7. San Venceslao, Duque de Bohemia. † 935.- Sin datos de canonización. Patrón de Bohemia desde el S. XI. 